\title{
Fractional Lengths and Crossing Numbers ${ }^{\star}$
}

\author{
Ondrej Sýkora ${ }^{1}$, László A. Székely ${ }^{2}$, and Imrich Vrto ${ }^{3}$ \\ 1 Department of Computer Science, Loughborough University \\ Loughborough, Leicestershire LE11 3TU, The United Kingdom \\ 2 Department of Mathematics, University of South Carolina \\ Columbia, SC 29208, USA \\ 3 Institute of Mathematics, Slovak Academy of Sciences \\ Dúbravská 9, 84235 Bratislava, Slovak Republic
}

\begin{abstract}
Adamec and Nešetřil [1] proposed a new the so called fractional length criterion for measuring the aesthetics of (artistic) drawings. They proposed to apply the criterion to the aesthetic drawing of graphs. In the graph drawing community, it is widely believed and even experimentally confirmed that the number of crossings is one of the most important aesthetic measures for nice drawings of graphs [6]. The aim of this note is to demonstrate on two standard graph drawing models that in provably good drawings, with respect to the crossing number measure, the fractional length criterion is closely related to the crossing number criterion.
\end{abstract}

\section{Introduction}

Adamec and Nešetřil 1 proposed a new the so called fractional length criterion for measuring the aesthetics of (artistic) drawings. A drawing $D$ is a finite set of continuous rectifiable curves in the Euclidean plane. The fractional length of the drawing $D$, is defined as follows. For a line $l$ denote by $\mathrm{i}(l, D)$ the number of intersections of the line $l$ and the drawing $D$. The fractional length $\mathrm{fl}(D)$ of the drawing $D$ is the expected value of $\mathrm{i}(l, D)$, where the expectation relates to the random selection of $l$ such that $l$ has a nonempty intersection with the convex hull of $D$. See Fig. 1 and Fig. 2 The fractional length of the second drawing is roughly 4.81 .

The fractional length is invariant under transposition, rotation and scaling. The criterion is also applicable to aesthetic graph drawings. See Fig. 2, In the graph drawing community, it is widely believed and even experimentally confirmed that the number of crossings is one of the most important aesthetic measures for nice drawing of graphs 36 . The aim of this note is to demonstrate on two standard graph drawing models that in provably good drawings, with respect to the crossing number measure, the fractional length criterion is closely related to the crossing number criterion. Under provably good drawing we understand a drawing in which the number of crossings differs from the minimum

* This research was supported in part by the EPSRC grant GR/R37395/01, NSF contract 0072187 and the VEGA grant 2/7007/20.

M.T. Goodrich and S.G. Kobourov (Eds.): GD 2002, LNCS 2528, pp. 186-192, 2002.

(C) Springer-Verlag Berlin Heidelberg 2002 


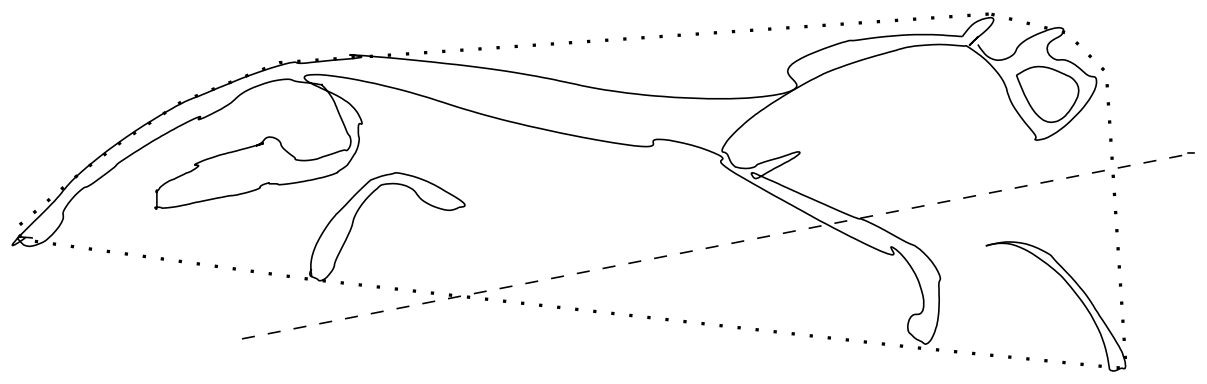

Fig. 1. A prehistoric drawing - the Uffington white horse.

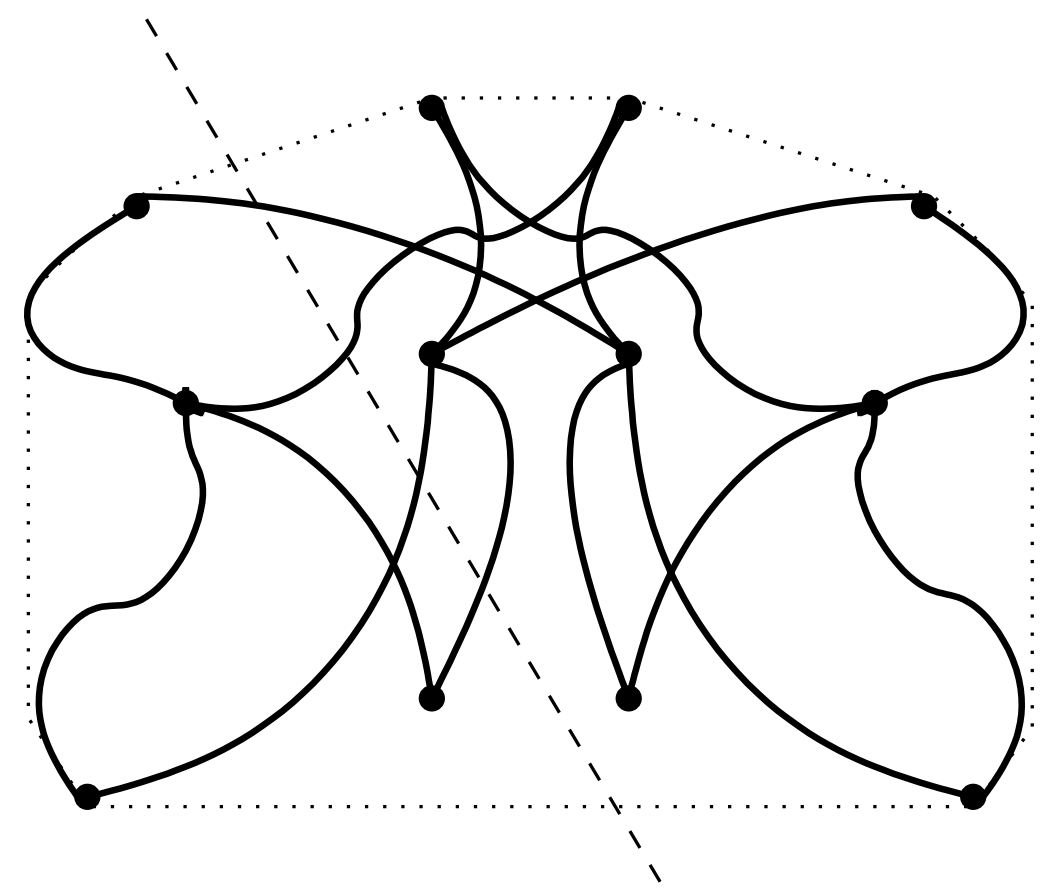

Fig. 2. A drawing of the 2-dimensional ordinary Butterfly graph inspired by the web page http://www. uni-paderborn.de/cs/ag-madh/.

by a polylogarithmic factor. More precisely, in the orthogonal model of drawing the relation between both parameters is almost quadratical while in the 2-layer drawing model is linear. 


\section{Definitions}

Let $G=(V, E)$ be a graph. Let $\operatorname{cr}(D)$ denote the number of crossings of edges in a drawing $D$ of $G$ in the plane. The crossing number of the graph $G$, denoted by $\operatorname{cr}(G)$, is the minumum of $\operatorname{cr}(D)$ over all drawings of $G$.

Let $G$ be a connected graph of maximum degree of at most 4 . Consider an infinite grid of horizontal and vertical lines with unit distances between any two parallel neighbouring lines. The VLSI layout for the graph $G$ is an injective mapping of vertices of $G$ into nodes of the grid and a mapping of the edges of $G$ into paths of the grid such that two paths can cross or touch at the grid nodes but can not overlap. See Fig. 3. The area $A$ of $G$ is the area of the smallest rectangle covering the layout, where the minumum is taken over all VLSI layouts of $G$ in the grid [9]. Note that any VLSI layout of $G$ produces the so called orthogonal layout.

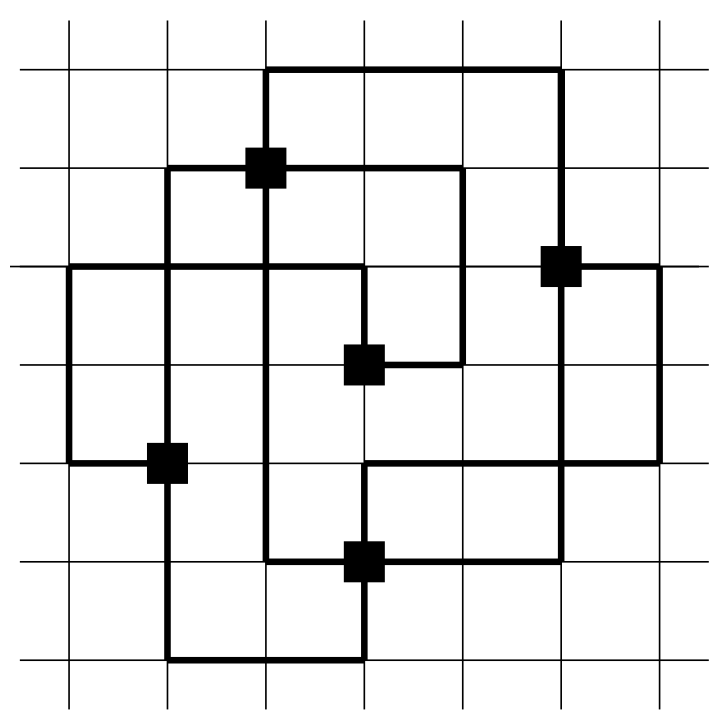

Fig. 3. A VLSI layout of a graph within area 49.

Let $G=\left(V_{0}, V_{1}, E\right)$ be a bipartite graph. Place the vertices of $V_{0}$ and $V_{1}$ into distinct points on horizontal lines $y=0$ and $y=1$, in the standard coordinate systems, respectively. Draw the edges by straight line segments producing a 2layer drawing $D$ of $G$. See Fig. 4 Let $\operatorname{bcr}(D)$ denote the number of crossings of edges of $D$. The bipartite crossing number of $G$ is the minimum of $\operatorname{bcr}(D)$ over all drawings $D$ of $G$. 
For a graph $G=(V, E)$, let $f:|V| \rightarrow\{1,2, \ldots,|V|\}$ be a bijection. The optimal linear arrangement problem for $G$ is to find

$$
\operatorname{Length}(G)=\min _{f} \sum_{u v \in E}|f(u)-f(v)| .
$$

Now we introduce the main tool for estimating fractional lengths of drawings. Steinhaus' paper [8] implies the following lemma:

Lemma 1. Consider a finite system of rectifiable curves in the plane of total length $L$. Let $P$ be the perimeter of the convex hull of the corresponding drawing D. Then

$$
\mathrm{fl}(D)=\frac{2 L}{P}
$$

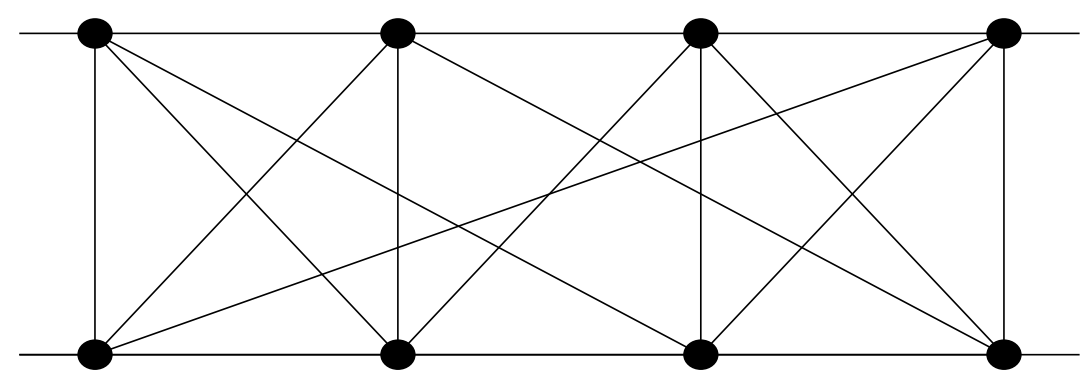

Fig. 4. A 2-layer drawing of the 3-dimensional hypercube.

\section{VLSI Model}

Our first drawing model with provably good crossing numbers is the orthogonal graph drawing based on the VLSI layout of graphs.

\subsection{Basic Result}

Theorem 1. Let $G=(V, E)$ be an $n$-vertex graph with the maximum degree of at most 4. Consider a VLSI layout of $G$ with the area $A$. Then there exists an orthogonal drawing $D$ of $G$ with a provably good number of crossings $\operatorname{cr}(D)$ and positive constants $c_{1}$ and $c_{2}$ satisfying

$$
c_{1} \frac{\sqrt{\operatorname{cr}(D)+n}}{\log n} \leq \mathrm{f}(D) \leq c_{2} \sqrt{\operatorname{cr}(D)+n} \log n .
$$

Proof. As we do not care about the constant factors we may assume that the optimal VLSI layout of $G$ lies in a square. In fact, Leiserson [5] proved that any 
layout lying in a rectagle of the area $A$ can be redrawn by "folding" in such a way that it occupies a square of the area $A^{\prime} \leq 3 A$. The optimal VLSI layout of $G$ produces in a natural way an orthogonal drawing $D$ of $G$. The results of Leighton [4, Leiserson [5] and Valiant [10] implies:

$$
\operatorname{cr}(G)+n \leq \operatorname{cr}(D)+n \leq A \leq c_{3}(\operatorname{cr}(G)+n) \log ^{2} n \leq c_{3}(\operatorname{cr}(D)+n) \log ^{2} n,
$$

for some constant $c_{3}$. This means that the drawing induced by the optimal VLSI layout produces a provably good drawing as it provides a polylogarithmic approximation of the crossing number of $G$. Let $L$ be the sum of lengths of all edges of $G$ in the layout. Observe that

$$
\frac{1}{2}(\operatorname{cr}(D)+n) \leq L \leq 2 A .
$$

In fact, horizontal or vertical straightline segments of lenght $1 / 2$ starting at a single vertex or crossing in the drawing contribute to $L$ by at least $1 / 2$. To see the second inequality, take a unit square and move its center along all edges in the drawing. The total area of covered points is no greater then $A$, but every point is covered at most twice.

Now consider our drawing as a union of curves in the plane of total length $L$ with a square convex hall of the area $A$. According to the Lemma 1 we have

$$
\mathrm{fl}(D)=\frac{L}{2 \sqrt{A}} .
$$

From (1) and (2) we get

$$
\frac{\operatorname{cr}(D)+n}{4 \sqrt{A}} \leq \frac{L}{2 \sqrt{A}} \leq \sqrt{A} \leq \sqrt{c_{3}} \sqrt{\operatorname{cr}(D)+n} \log n .
$$

Hence

$$
\frac{1}{\sqrt{c_{3}}} \frac{\sqrt{\operatorname{cr}(D)+n}}{\log n} \leq \frac{L}{2 \sqrt{A}} \leq \sqrt{c_{3}} \sqrt{\operatorname{cr}(D)+n} \log n .
$$

Finally, substituting (3) into (4) we get the result.

Our results roughly say that the fractional length of a provably good drawing, with respect to crossing number measure, is proportional to the square root of the number of crossings plus the number of vertices. It suggests that the fractional length expresses for orthogonal drawings the same "beauty" as the crossing number but measured in different units. Note that it is impossible to remove the $n$ term in $\operatorname{cr}(D)+n$, because an $\sqrt{n} \times \sqrt{n}$ mesh graph has crossing number zero but the fractional lenght is of order $\sqrt{n}$. This suggests that the aesthetic measure $\operatorname{cr}(\mathrm{G})+n$ is more suitable than just a sole $\operatorname{cr}(G)$.

We compared the fractional length measure with the number of crossings in "good" drawings with small number of crossings. A comparison to any drawing would be more desirable but one can easily see that if we consider a general drawing model in the plane then any drawing of a graph can be redrawn keeping the number of crossings same but increasing the fractional length arbitrarily. So without putting "reasonable" assumption on the drawing the both measures are incoparable. 


\section{Two-Layer Model}

Our second model of provably good graph drawing is the 2-layer model with the bipartite crossing number measure.

Consider an $n$-vertex bipartite graph $V=\left(V_{0}, V_{1}, E\right)$. As the bipartite crossing number depends on the orderings of vertices on the lines $y=0$ and $y=1$ only, we may assume that the vertices are placed into points with integer coordinates such that the whole drawing fits into an $(n-1) \times 1$ rectangle.

\subsection{General Graphs}

Theorem 2. Let $G=\left(V_{0}, V_{1}, E\right)$ be an $n$-vertex bipartite graph of minimum and maximum degree $\delta$ and $d$. Let $\delta \geq 2$ and $d=O(\delta)$. Then there exists a 2-layer drawing $D$ of $G$ with a provably good number of crossings $\operatorname{bcr}(D)$ and positive constants $c_{1}, c_{2}$ and $c_{3}$ satisfying

$$
c_{1} \frac{1}{d n} \operatorname{bcr}\left(D \leq \mathrm{fl}(D) \leq c_{2} \frac{1}{d n} \operatorname{bcr}(D)+c_{3} d .\right.
$$

Proof. Consider an optimal linear arrangement of $G$. We may assume that the vertices of $G$ are placed on the line $y=0$ into points $\{1,2, \ldots, n\}$. Projecting orthogonally the vertices of $V_{1}$ on the line $y=1$ and drawing the edges of $G$ as straight line segments we obtain a 2-layer drawing $D$ of $G$. The results of Shahrokhi et al. 7] implies

$$
\operatorname{bcr}(G) \leq \operatorname{bcr}(D) \leq 5 d \text { Length }(G)
$$

and

$$
c_{4} d \operatorname{Length}(G) \leq \operatorname{bcr}(G)+\frac{1}{12} n d^{2} \leq \operatorname{bcr}(D)+\frac{1}{12} n d^{2},
$$

for some positive constant $c_{4}$. The above inequalities immediately imply that the bipartite crossing number of the drawing $D$ is approximately the same as the optimal value $\operatorname{bcr}(G)$. Now consider our drawing $D$ as a union of curves in the plane of total length $L$ with a rectangular convex hall of the perimeter $2 n$. Clearly

$$
\operatorname{Length}(G) \leq L \leq \sqrt{2} \operatorname{Length}(G) .
$$

Finally, combining (5), (6) and (7) and noting that $\mathrm{fl}(D)=L / n$, we get

$$
\frac{\operatorname{bcr}(D)}{5 d n} \leq \mathrm{fl}(D) \leq \frac{\sqrt{2} \mathrm{bcr}(D)}{c_{4} d n}+\frac{\sqrt{2}}{c_{4}} d
$$

which implies the claim.

\subsection{Trees}

A more precise relation between the fractional length and the bipartite crossing number holds in the case of trees. 
Theorem 3. Let $T$ be an $n$-vertex tree and $d_{v}$ denote the degree of any vertex $v$. Then there exist a 2-layer optimal drawing $D$ of $T$ satisfying

$$
\begin{aligned}
& \mathrm{fl}(D) \geq \frac{1}{n}\left(\operatorname{bcr}(D)+n-1+\sum_{v \in T}\left\lceil\frac{d_{v}}{2}\right\rceil\left\lfloor\frac{d_{v}}{2}\right\rfloor\right) \\
& \mathrm{fl}(D) \leq \frac{\sqrt{2}}{n}\left(\operatorname{bcr}(D)+n-1+\sum_{v \in T}\left\lceil\frac{d_{v}}{2}\right\rceil\left\lfloor\frac{d_{v}}{2}\right\rfloor\right)
\end{aligned}
$$

Proof. Shahrokhi et al. 7] proved that an optimal 2-layer drawing $D$ of a tree $T$ can be obtained from an optimal linear arrangemment of $T$ by the above projection and moreover

$$
\operatorname{bcr}(T)=\operatorname{bcr}(D)=\operatorname{Length}(T)+1-n-\sum_{v \in T}\left\lceil\frac{d_{v}}{2}\right\rceil\left\lfloor\frac{d_{v}}{2}\right\rfloor .
$$

The rest of the proof is similar as above.

\section{References}

1. Adamec, J., Nešetřil, J.: Towards an Aesthetic Invariant for Graph Drawing. In: 9th Intl. Symp. on Graph Drawing. LNCS 2265. Springer. Berlin (2001) 287-296

2. Bhatt, S., Leighton, F.T.: A Framework for Solving VLSI Graph Layout Problems. J. Computer and System Science 28 (1984) 300-334

3. DiBattista, G., Eades, P., Tollis, I., Tamassia, R.: Graph Drawing: Algorithms for the Visualization of Graphs. Prentice Hall (1999)

4. Leighton, F.T.: New Lower Bound Techniques for VLSI. Mathematical Systems Theory 17 (1984) 47-70

5. Leiserson, C.E.: Area Efficient Graph Layouts (for VLSI). In: 21st Annual Symposium on Foundations of Computer Science. IEEE Press (1980) 270-281

6. Purchase, H.: Which aestethic has the greatest effect on Human Understanding? In: 5th Intl. Symp. on Draph Drawing. LNCS 1353. Springer. Berlin (1997) 248-261

7. Shahrokhi, F., Sýkora, O., Székely, L.A., Vrto, I.: On Bipartite Drawings and the Linear Arrangement Problem. SIAM J. Computing 30 (2000), 1773-1789.

8. Steinhaus, H.: Length, Shape and Area. Colloquium Math. III (1954) 1-13

9. Thompson, C.D.: Area-Time Complexity for VLSI. In: 11th Annual ACM Symposium on Theory of Computing. ACM Press (1979) 81-89

10. Valiant, L.G.: Universality Considerations in VLSI Circuits. IEEE Transactions on Computers 30 (1981) 135-140 